\title{
Promoting Robust Design of Diode Lasers for Space: A National Initiative
}

\author{
David M. Tratt', Mark A. Stephen², Farzin Amzajerdian³, \\ Nasir B. Kashem², Andrew A. Shapiro ${ }^{4}$, and Allan T. Mense
}

1 The Aerospace Corporation, Pasadena, CA 91101.

2 NASA Goddard Space Flight Center, Greenbelt, MD 20771.

3 NASA Langley Research Center, Hampton, VA 23681.

4 Jet Propulsion Laboratory, California Institute of Technology, Pasadena, CA 91109.

${ }^{5}$ Raytheon Company, Tucson, AZ 85706. 


\section{Background}

- The NASA Earth Science Technology Office sponsored an industry/USG Community Forum on Laser Diode Arrays in Space-Based Applications March 2-3, 2004 in Arlington, Va.

- Attendance: 23 industry, 42 USG/contractors, 6 other.

- Purpose \#1: Brief 2003 ICESat/GLAS on-orbit laser failure to community.

- Purpose \#2: Intensify dialog between LDA vendor and user communities concerning technology development requirements for robust long-life diode arrays suitable for deployment in space.

- Frank debate between USG and industry concerning each others' needs.

- NASA established the Diode-laser Array Working Group in July of 2004 to continue the dialog.

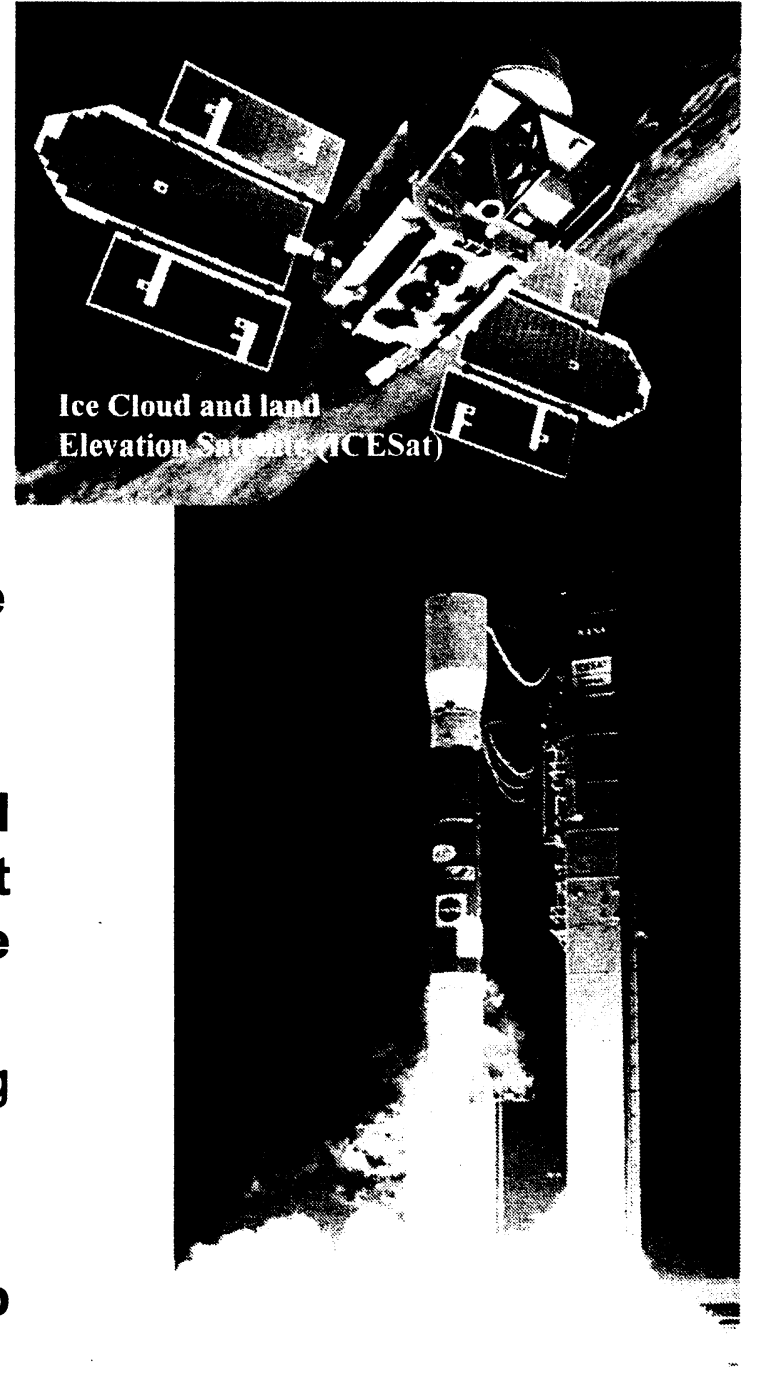




\section{Working Group Charter: Purpose}

- Provide a national-level consumer/provider forum for discussion of engineering and manufacturing issues which impact the reliability and survivability of high-power broad-area laser diodes and laser diode arrays (LDAs) in space.

- Formulate and validate standardized test protocols and derating recommendations by reference to test data.

- Recommend USG investment and procurement strategies for assuring the quality and availability of space-qualified LDAs.

- Facilitate collaboration between stakeholder USG entities (NASA, DOD, DOE), aerospace system integrators, and laser diode suppliers.

- Provide standardized guidelines for diode laser vendors and programs developing and qualifying parts for space-flight applications. 


\section{Working Group Charter: Scope}

- Review practices and lessons learned from past, current and planned activities among the Group members.

- Evaluate current and proposed design and engineering practices as they relate to component reliability.

- Synthesize and specify baseline environmental requirements that reflect the minimum qualification standards for laser diodes to be used in space flight applications.

- Recommend standardized testing protocols for the qualification of laser diodes for space flight.

- Develop consensus manufacturing, operational environment control and certification standards for incorporation into contractual vehicles between vendors and the USG. 


\section{NASA Test Facilities}

- NASA operates LDA test facilities for 808- and 792-nm devices.

- Facilities were established as a key component of the NASA Laser Risk Reduction Program (LRRP) activity.

- Facilities used to characterize performance and conduct lifetest evaluation of products from multiple vendors under consistent set of test conditions.

- Operations will be extended to additional wavelengths. 


\section{NASA Test racilities}

- NASA operates LDA test facilities for 808- and 792-nm devices.

- Facilities were established as a key component of the NASA Laser Risk Reduction Program (LRRP) activity.

- Facilities used to characterize performance and conduct lifetest evaluation of products from multiple vendors under consistent set of test conditions.

- Operations will be extended to additional wavelengths. 


\section{LŨLÁt LDÁ Fïigntí Requirements}

\begin{tabular}{|ll|}
\hline Package: & G2 (two-bar array) \\
\hline Operational wavelength: & $\mathbf{8 0 8} \mathrm{nm}$ \\
\hline Repetition frequency: & $28 \mathrm{~Hz}$ \\
\hline Pulse current: & $70 \mathrm{~A}(\max 90 \mathrm{~A})$ \\
\hline Current pulse width: & $170 \mu \mathrm{s}(\max 220 \mu \mathrm{s})$ \\
\hline
\end{tabular}

- The laser must operate for one billion pulses in vacuum.

- The laser has two G2 LDAs.

o To increase reliability of the system, two laser units were built, each with a projected lifetime of one billion pulses.

- LDAs from two vendors were selected as potential candidates for the mission.

- One major difference between vendors: Vendor 1 used indium solder and vendor 2 used gold/tin solder.

${ }^{t}$ Lunar Orbiter Laser Altimeter (LOLA) is an instrument aboard the Lunar Reconnaissance Orbiter (LRO) scheduled for Launch in December, 2008 


\section{LOLA LDÁ Test́ Frotocoi}

- LOLA project procured 60 LDA (30 from each vendor)

- Characterized all arrays

- Burned-in all arrays (100M pulses, $\left.90 \mathrm{~A}, 100 \mathrm{~Hz}, 200 \mu \mathrm{sec}, 25^{\circ} \mathrm{C}\right)$ and characterized all arrays after burned in

- Based on characterization data - chose arrays to be used in laser assembly and spares (total 10/vendor)

- Randomly chose arrays for vacuum and air tests - a total of $12 / v e n d o r$ set aside for testing

- Started 24/7 vacuum and air automated tests

- Analyze data, correlate characterization and performance data

- Report on findings of tests at time of flight array delivery 


\section{LOLÂ T̃esting Âpproach}

- Use spare vendor to mitigate risks associated with manufacturing problems and defects

- Accelerated tests achieved full mission goal (in pulse count) prior to laser integration

- LDAs tested in flight-like environment and operating conditions

- Continuing performance tests to improve statistics and knowledge base.

LOLA Performance Test Matrix

\begin{tabular}{|c|c|c|c|c|c|}
\hline $\begin{array}{c}\text { Environ- } \\
\text { ment }\end{array}$ & $\begin{array}{c}\text { Operating } \\
\text { Conditions } \\
\text { (pulse } \\
\text { width=170us) }\end{array}$ & $\begin{array}{c}\text { Peak } \\
\text { Power } \\
\text { Rating }\end{array}$ & $\begin{array}{l}\text { Duty } \\
\text { Cycle }\end{array}$ & $\begin{array}{l}\text { Vendor } \\
1 \text { (\# of } \\
\text { LDA) }\end{array}$ & $\begin{array}{l}\text { Vendor } \\
2 \text { (\# of } \\
\text { LDA) }\end{array}$ \\
\hline \multirow{2}{*}{ Vacuum } & $\begin{array}{c}\text { Nominal } \\
28 \mathrm{~Hz}, 70 \mathrm{~A}\end{array}$ & $70 \%$ & $0.48 \%$ & 3 & 3 \\
\hline & $\begin{array}{c}\text { Accelerated } \\
150 \mathrm{~Hz}, 70 \mathrm{~A}\end{array}$ & $70 \%$ & $2.55 \%$ & 3 & 3 \\
\hline \multirow{2}{*}{ Air } & $\begin{array}{l}\text { Accelerated } \\
210 \mathrm{~Hz}, 70 \mathrm{~A} \\
\end{array}$ & $70 \%$ & $3.57 \%$ & 3 & 3 \\
\hline & $\begin{array}{l}\text { Full Rating } \\
155 \mathrm{~Hz}, 100 \mathrm{~A}\end{array}$ & $100 \%$ & $2.64 \%$ & 3 & 3 \\
\hline
\end{tabular}




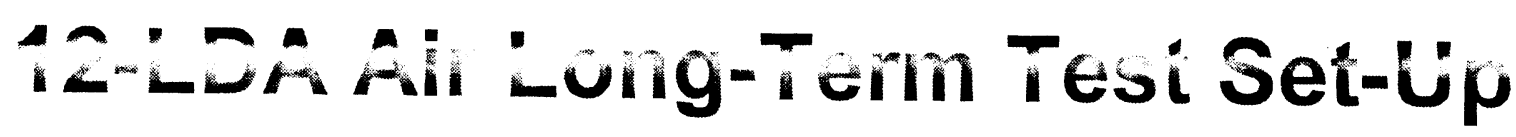
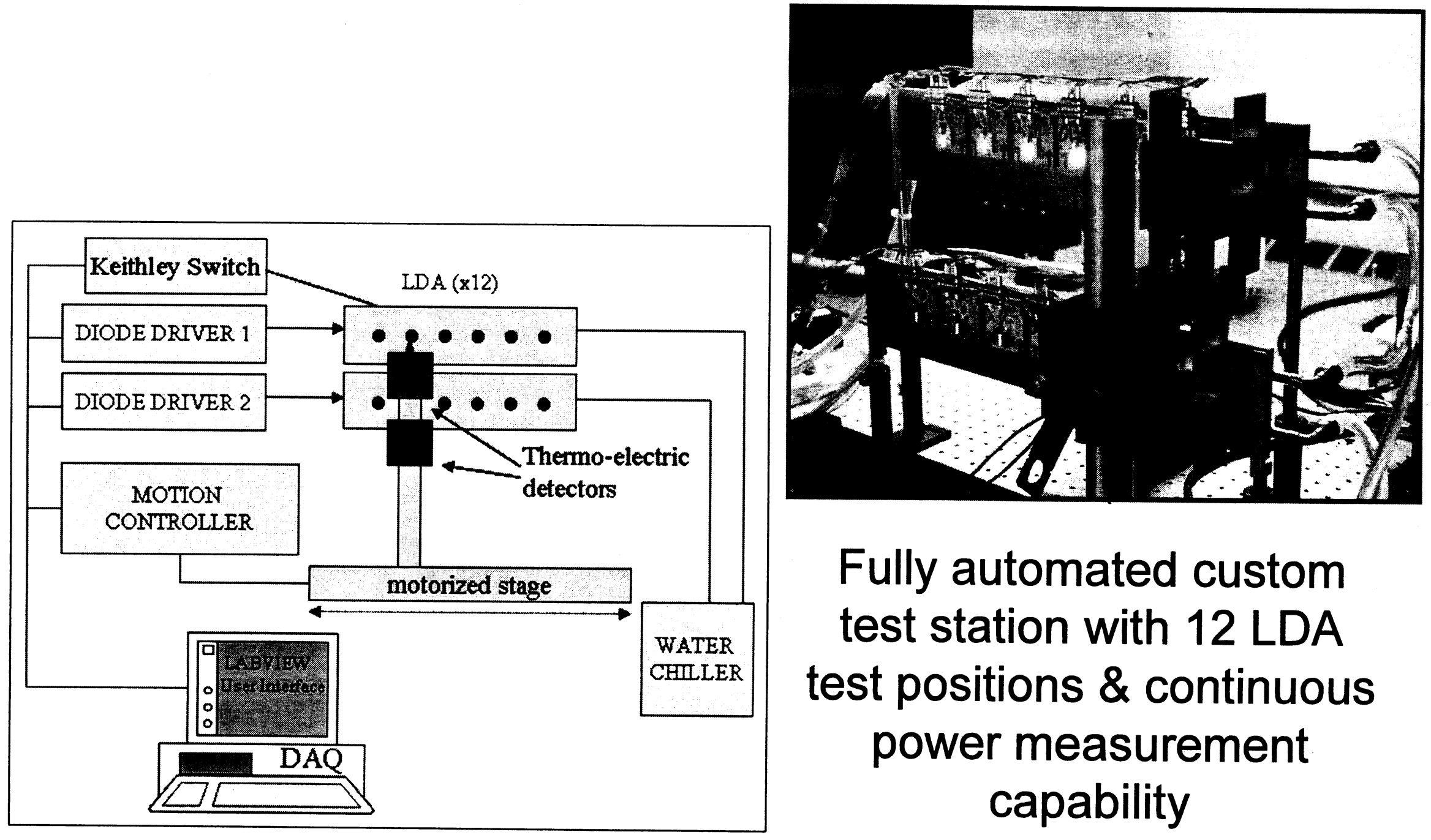

Fully automated custom test station with 12 LDA test positions \& continuous power measurement capability 


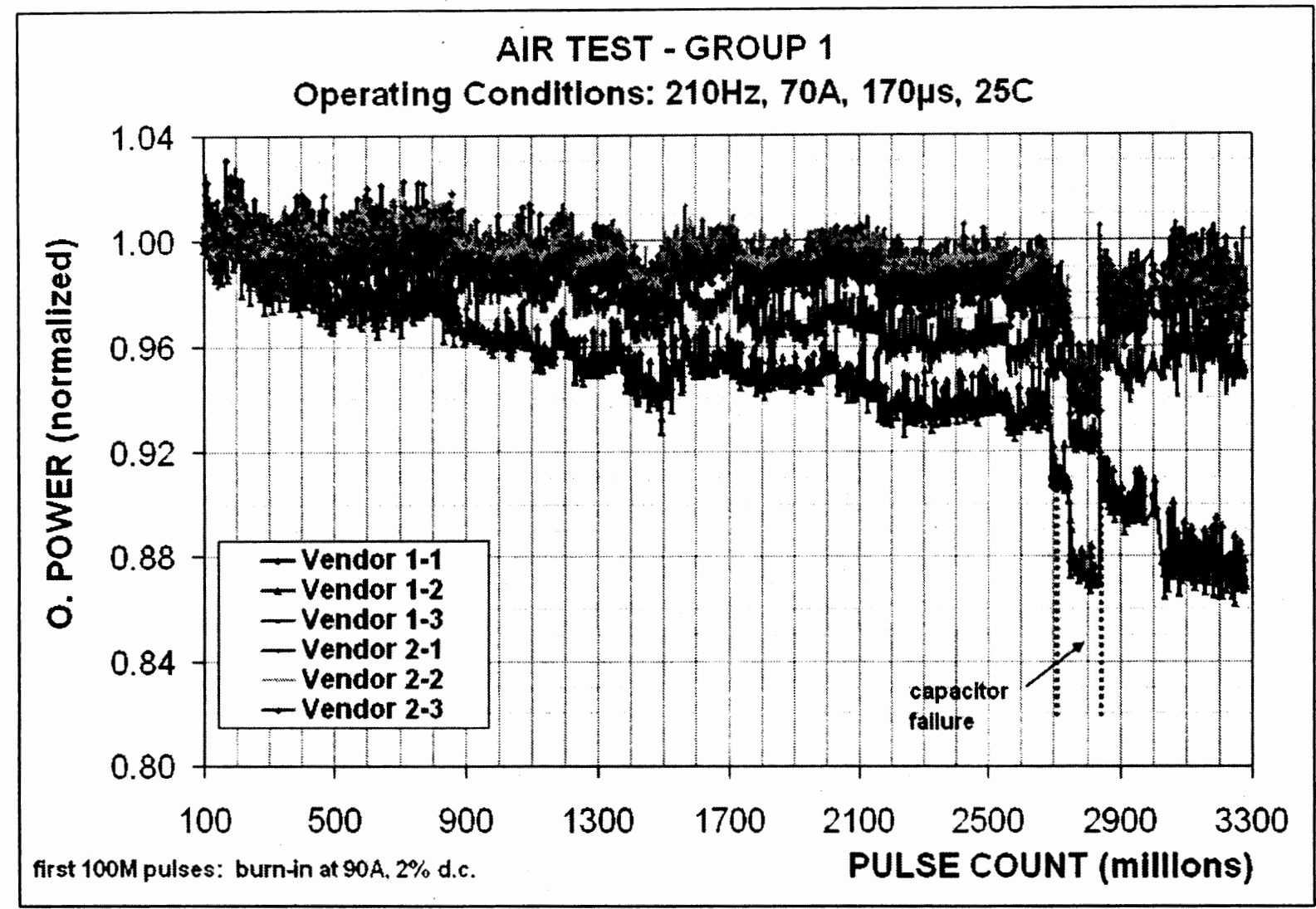

$>$ More than 3.2 billion accumulated pulses.

$>$ No bar failures.

$>$ One LDA (Vendor 1) shows significantly higher $(12 \%)$ power drop. 


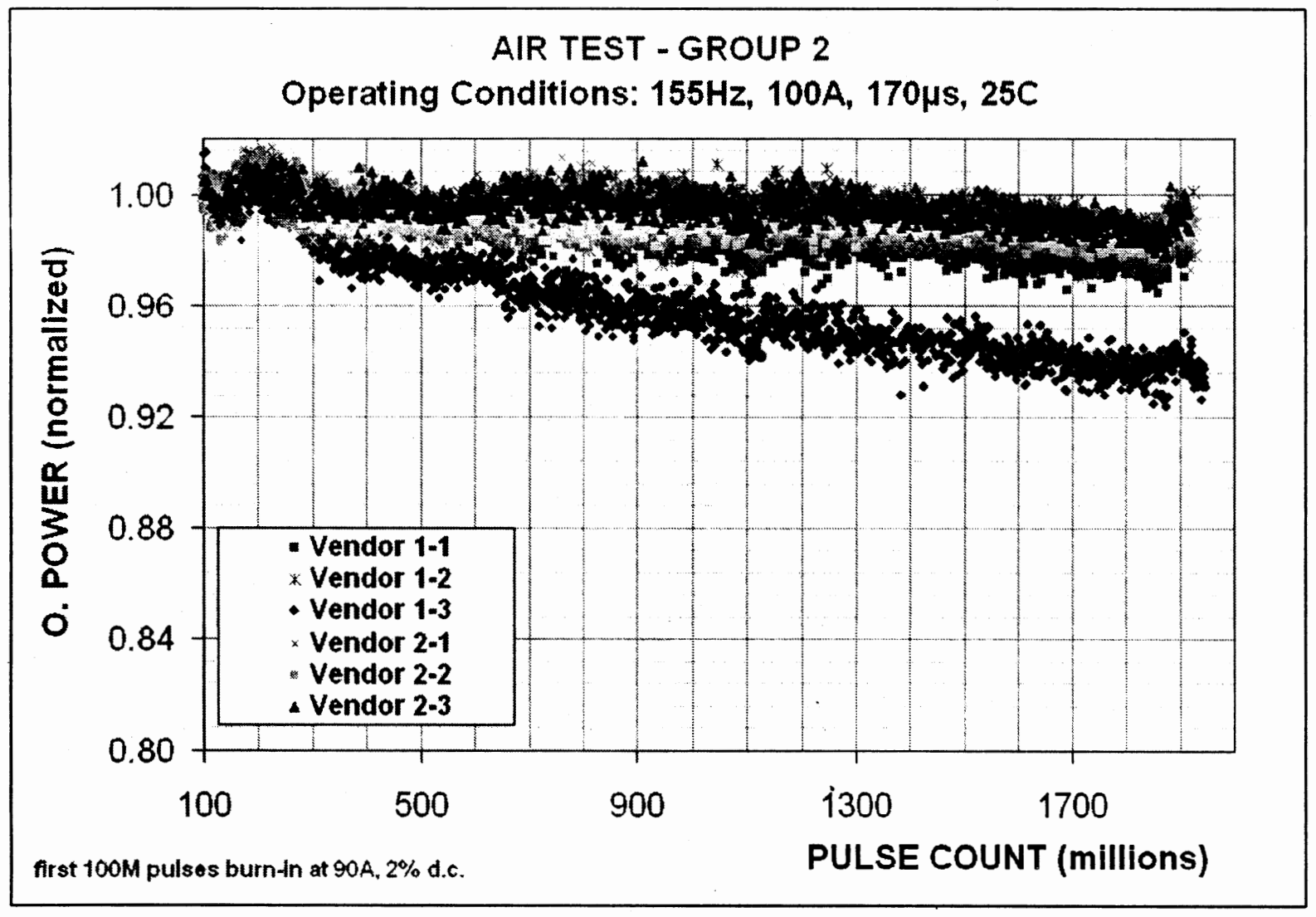

$>$ More than 1.9 billion accumulated pulses.

$>\quad$ No bar failures.

$>$ No significant degradation except for one LDA (Vendor 1). 


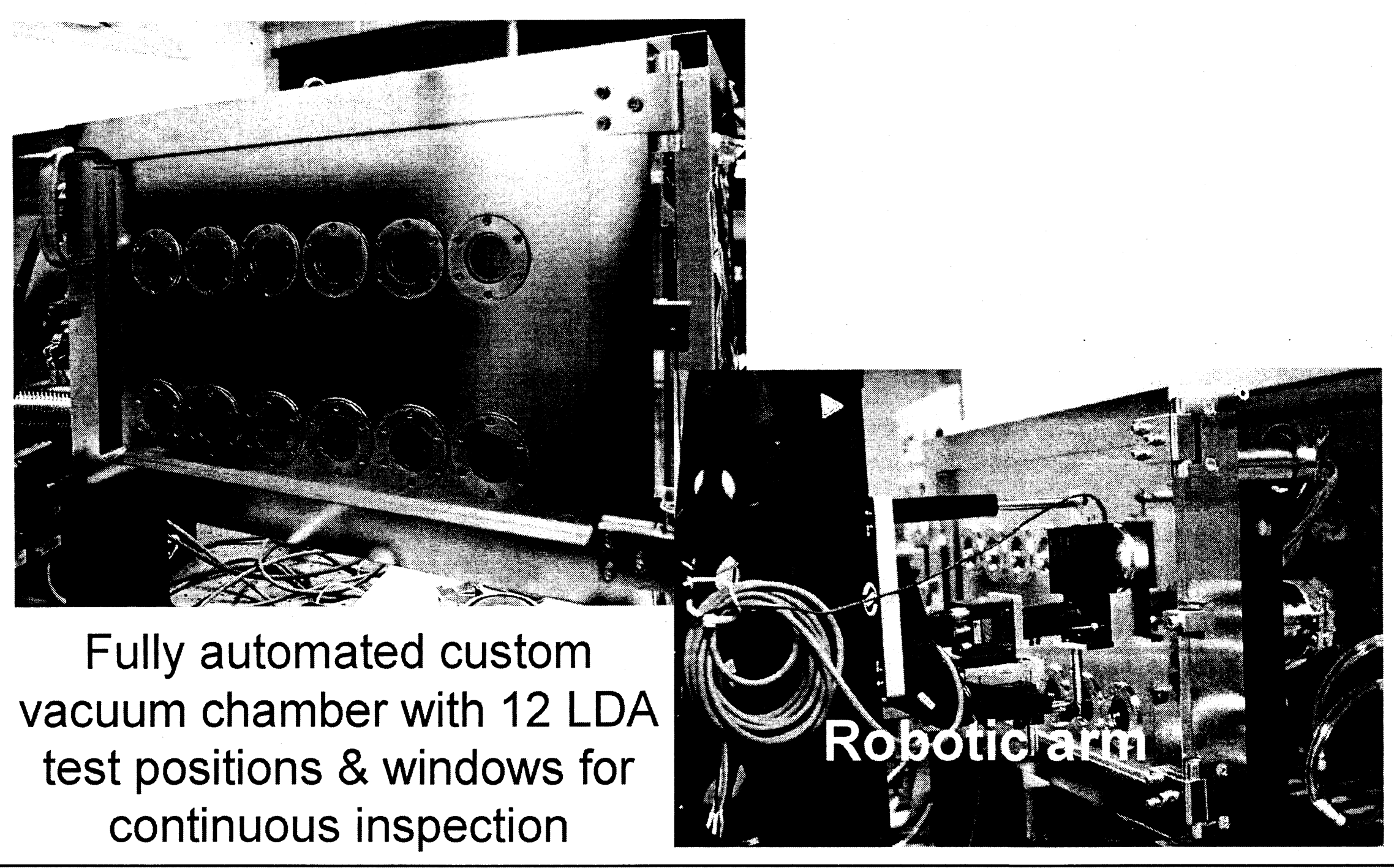




\section{LOLA Flight Lot Test in Vacuum}

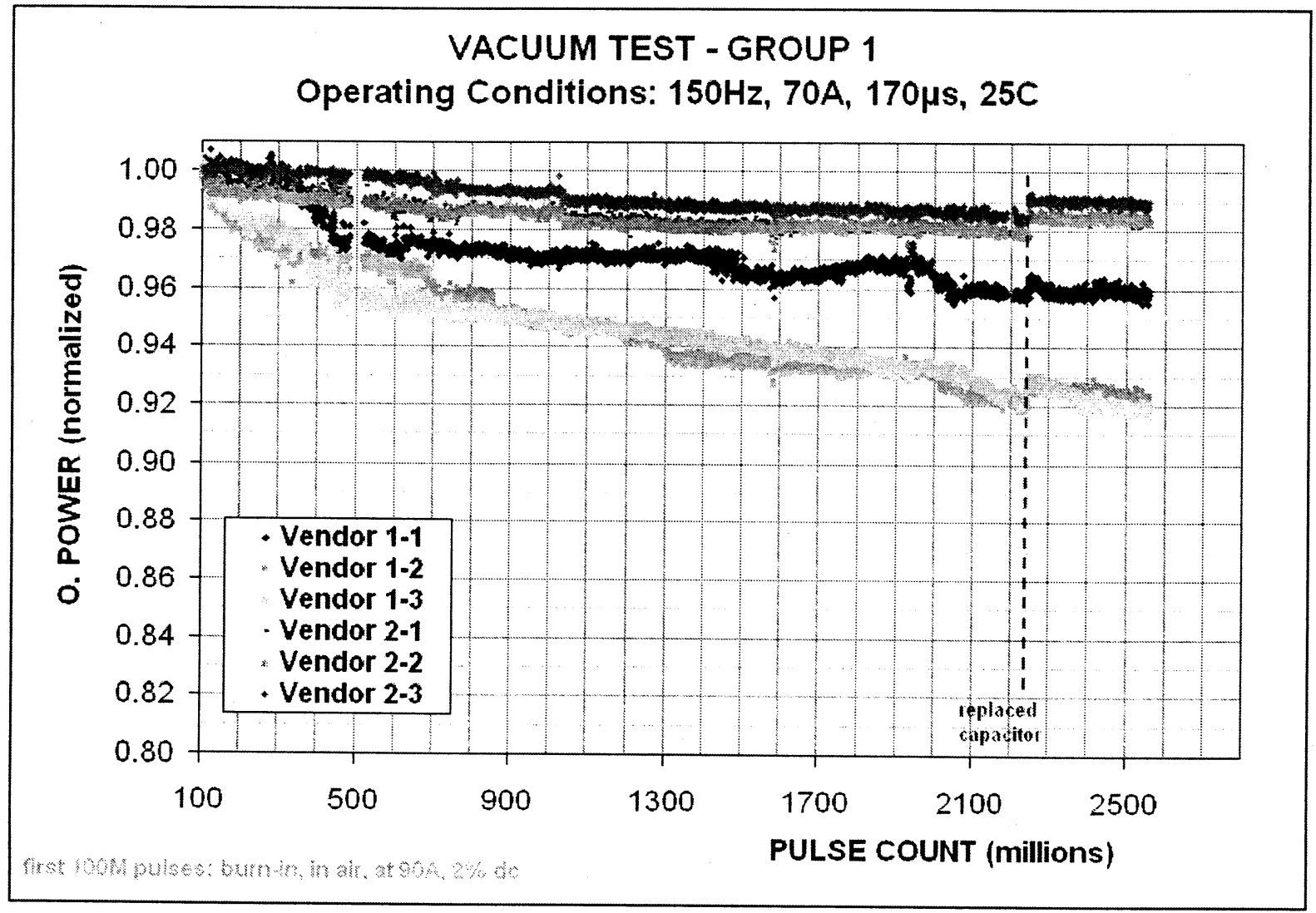

* More than 2.4 billion accumulated pulses.

* No bar failures.

* All LDA from Vendor 1 show reduction in optical power. 


\section{LOLA Flight Lot Test in Vacuum}

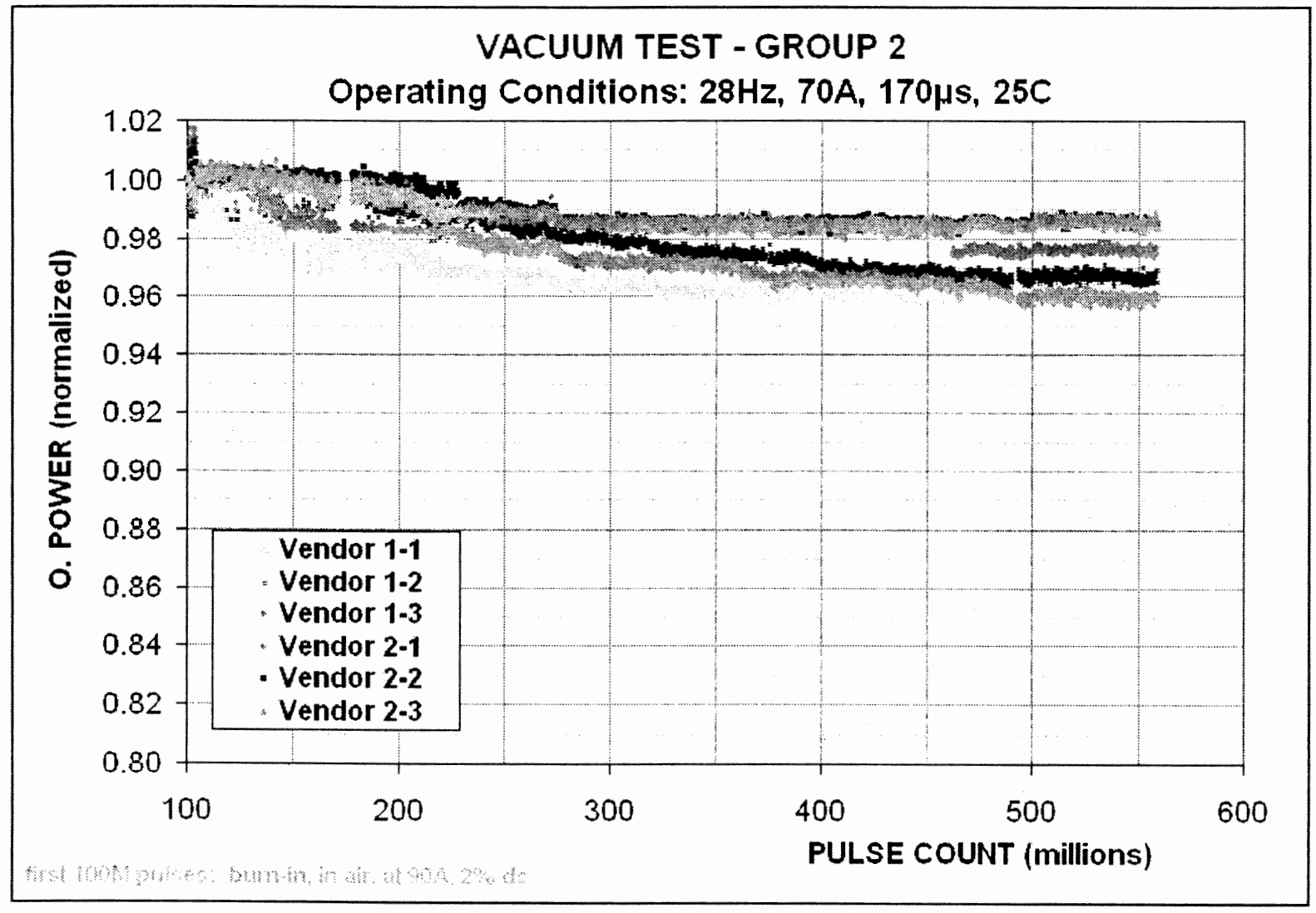

照
$>$ More than 500 million accumulated pulses.

$>$ No bar failures.

$>$ LDA from Vendor 1 show higher power decay rates.

$>$ Fluctuations in curves up to $200 \mathrm{M}$ pulses were due to test electronics. 


\section{2-nm Test Program at LaRC}

Focus is on lifetime and reliability of 792-nm LDAs operating in "Iong pulse duration mode" required for pumping 2-micron Tmand/or Ho-based lasers:

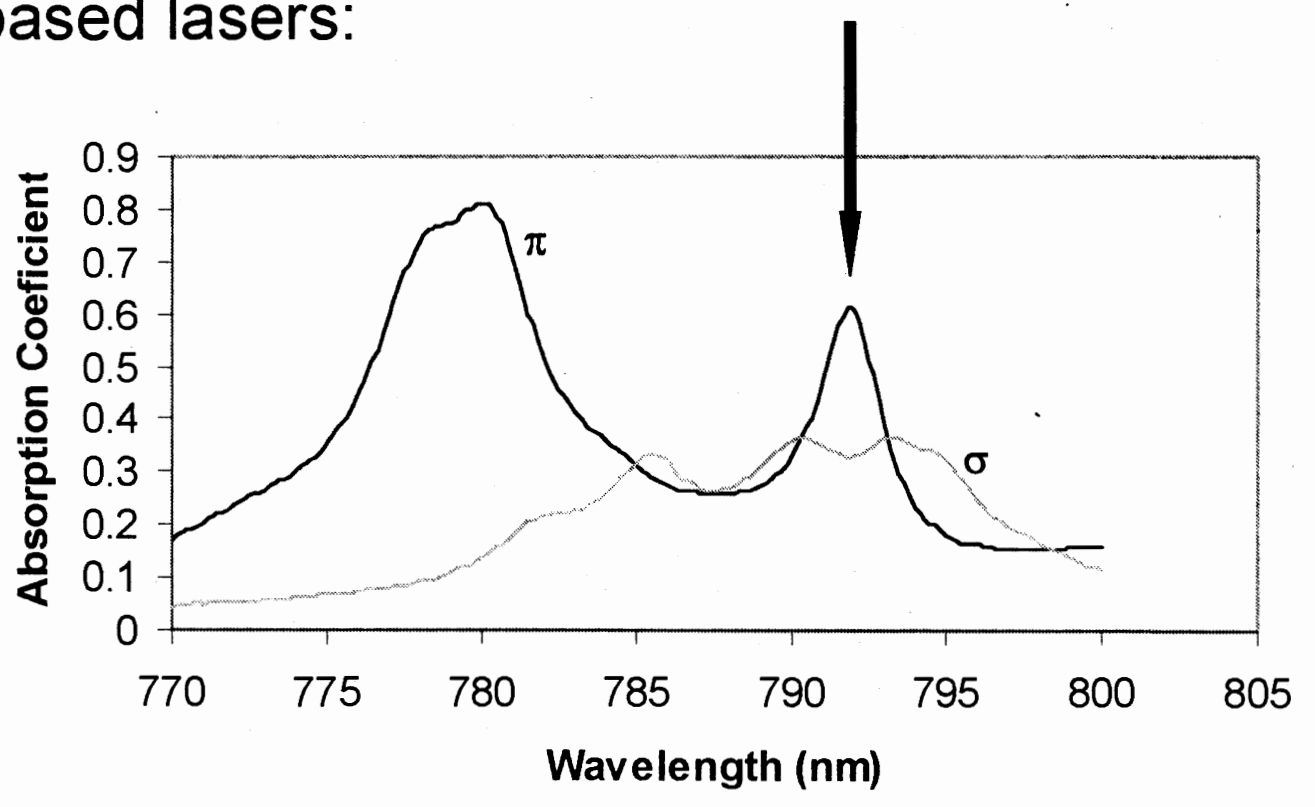

Operating in long pulse duration regime $(>1 \mathrm{msec}$ ) further limits the lifetime of laser diode arrays due to increased thermal stress. 


\section{2-nm Test Program at LaRC}

Visual Inspection
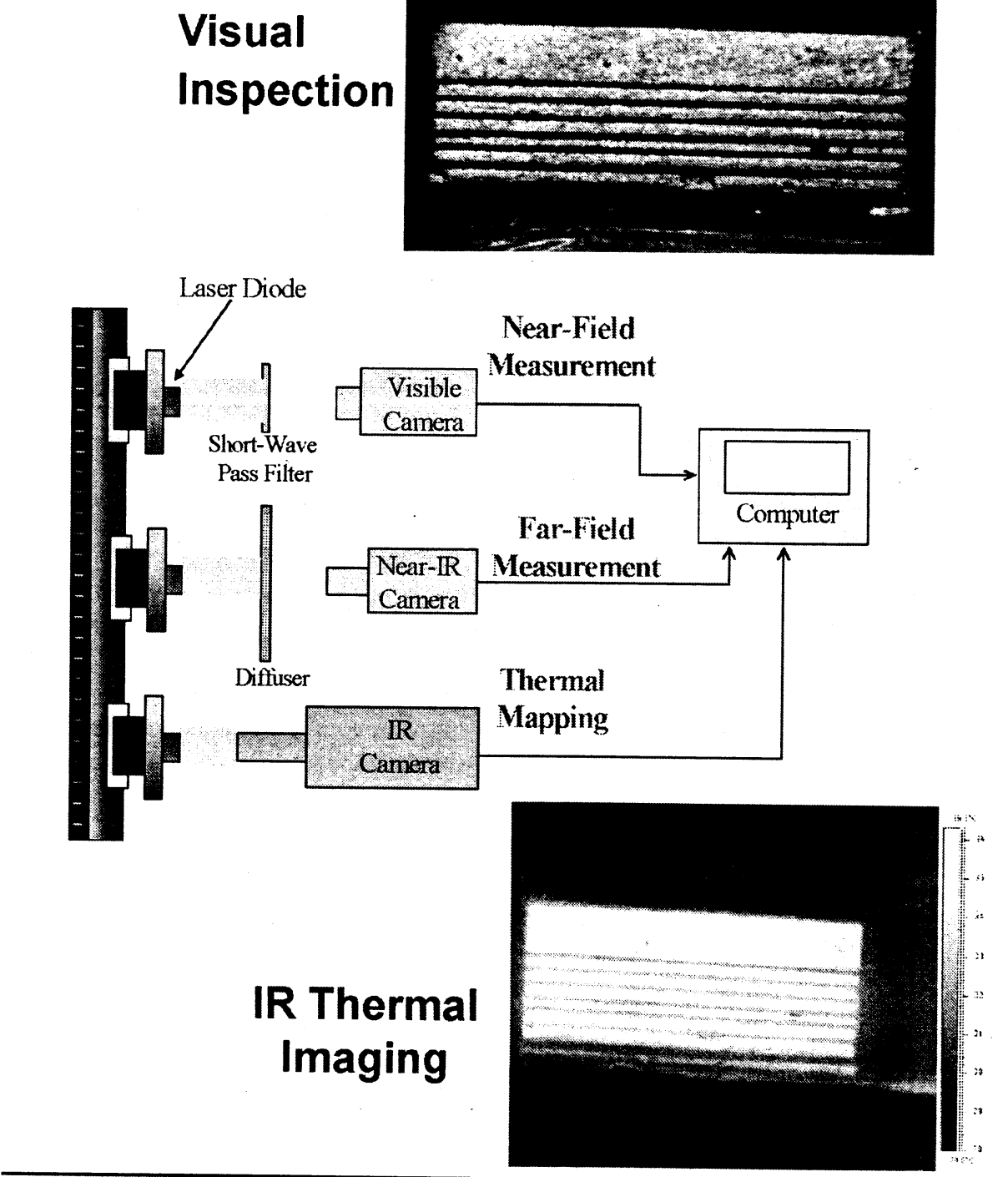

Far-Field

Measurement

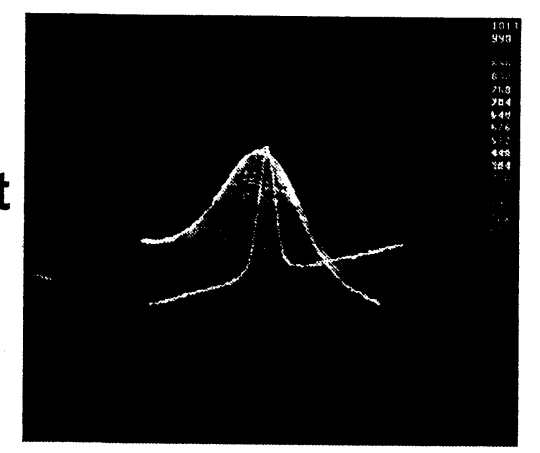

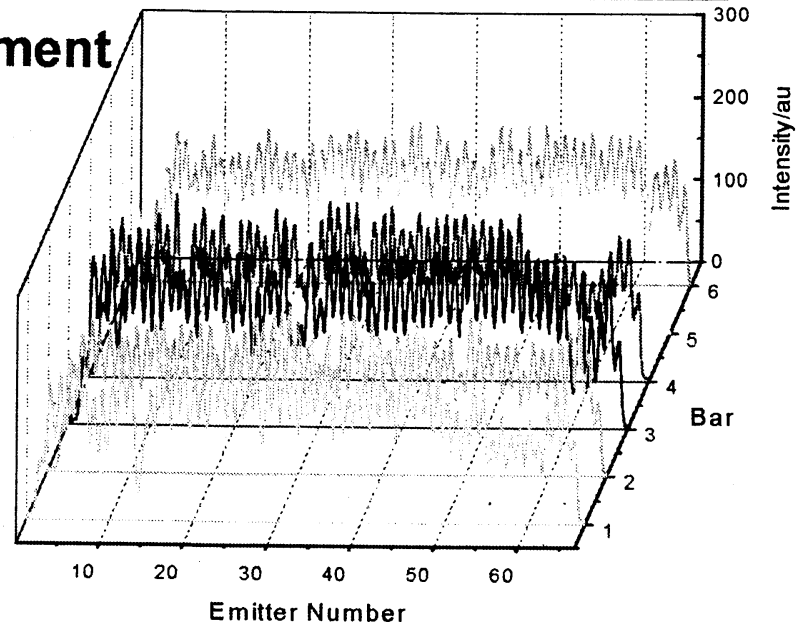




\section{2-nm Test Program at LaRC}

LaRC

IPI
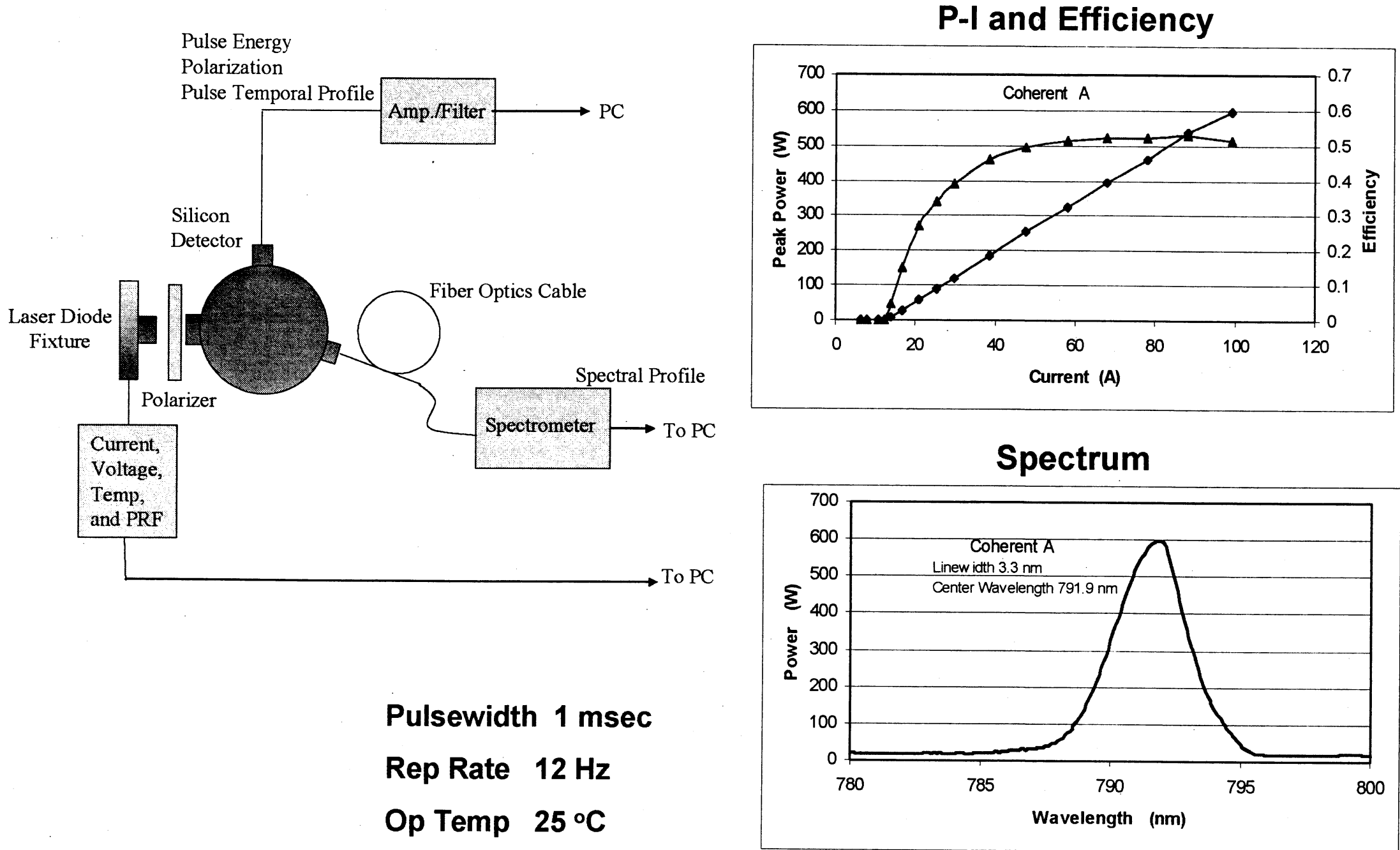

\section{Rep Rate $12 \mathrm{~Hz}$}

Op Temp $25^{\circ} \mathrm{C}$ 


\section{In-Depth Thermal Measurements}

IR Imaging

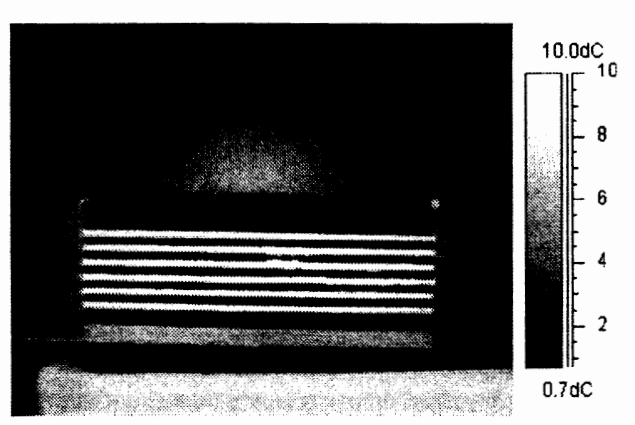

Temporally Resolved Spectral Measurement

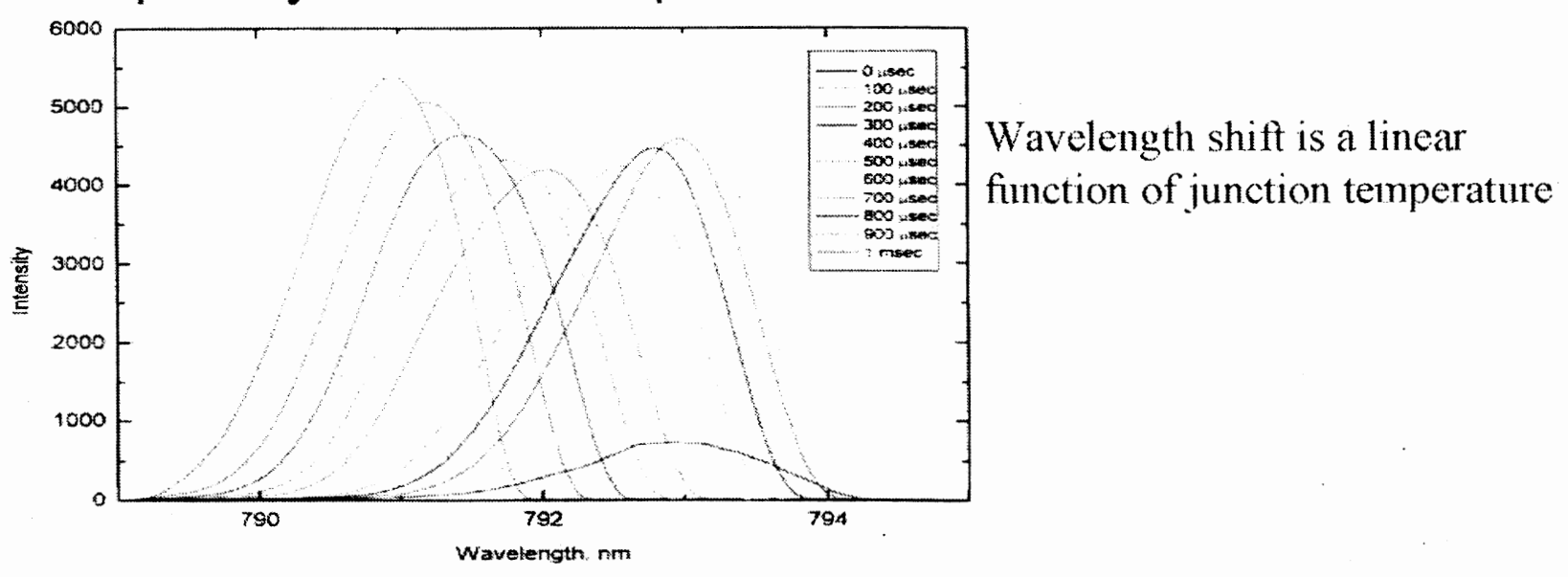

Forward Voltage-Short Pulse (Junction Temperature) Measurement

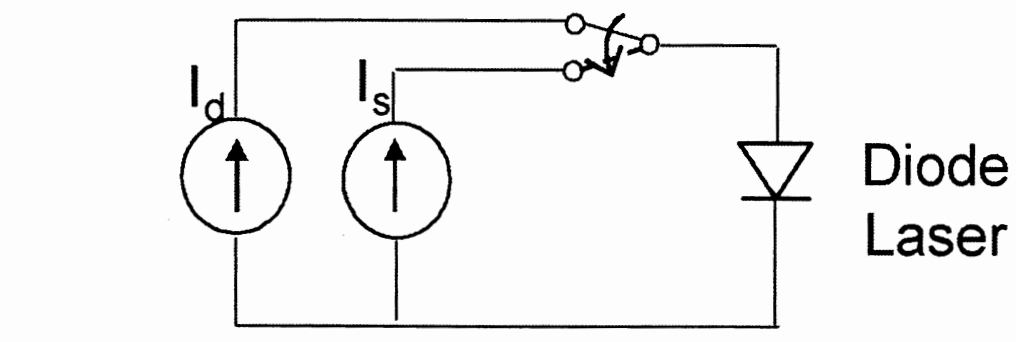
$83.3 \mathrm{msec}(12 \mathrm{~Hz})$

$1 \mathrm{msec}$

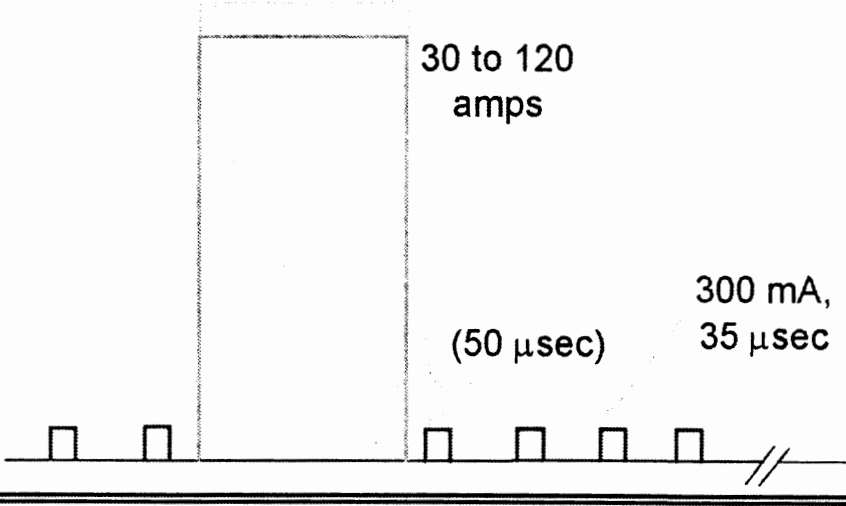




\section{Thermal Effects of QCW 792-nm LDA}

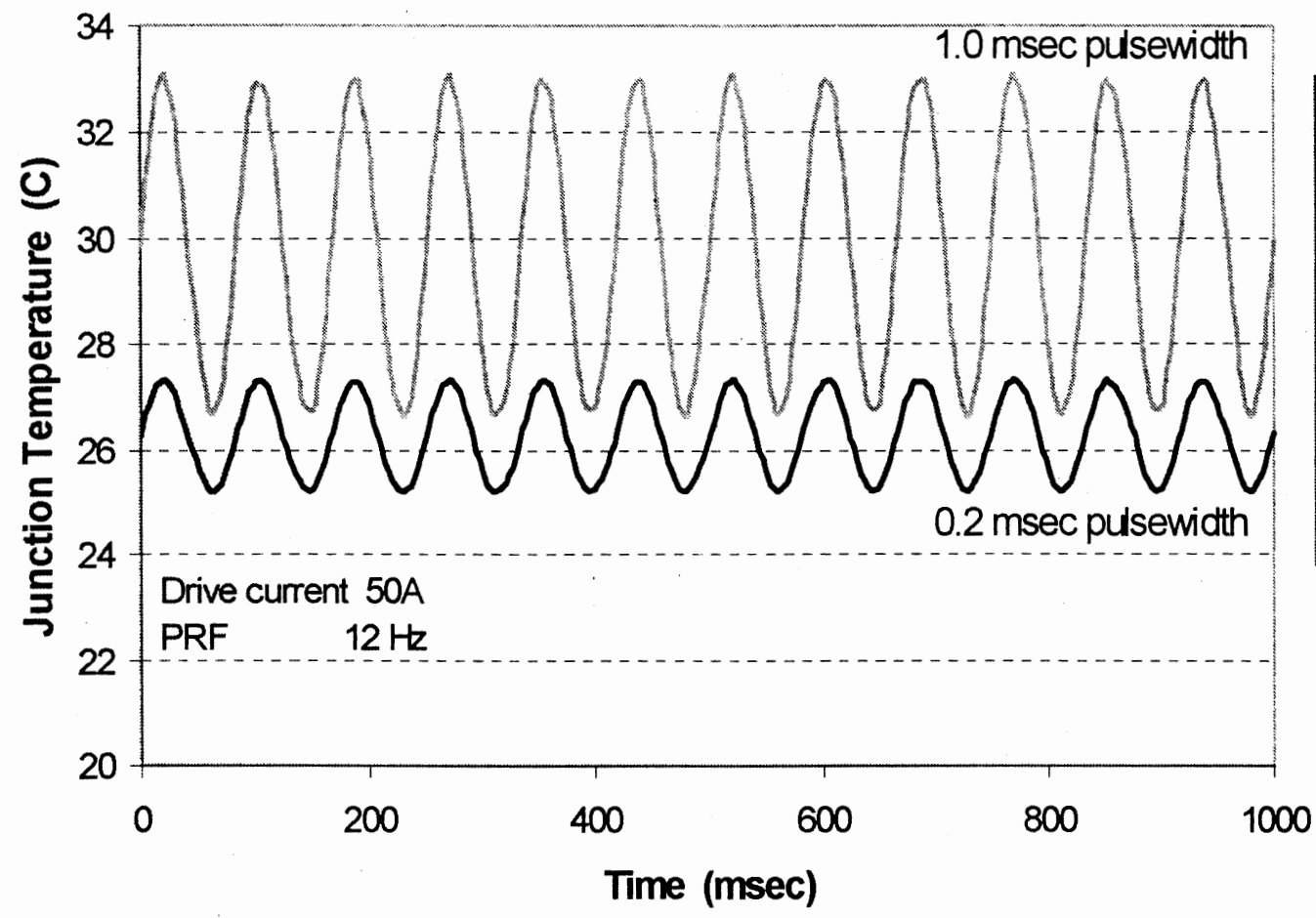

Excessive heating of the active region and drastic pulse-to-pulse thermal cycling limits the lifetime of laser diode arrays when operating in long pulse regime.

Lifetime models predict up to 2 orders of magnitude shorter lifetime when operating in $1 \mathrm{msec}$ pulsewidth regime 


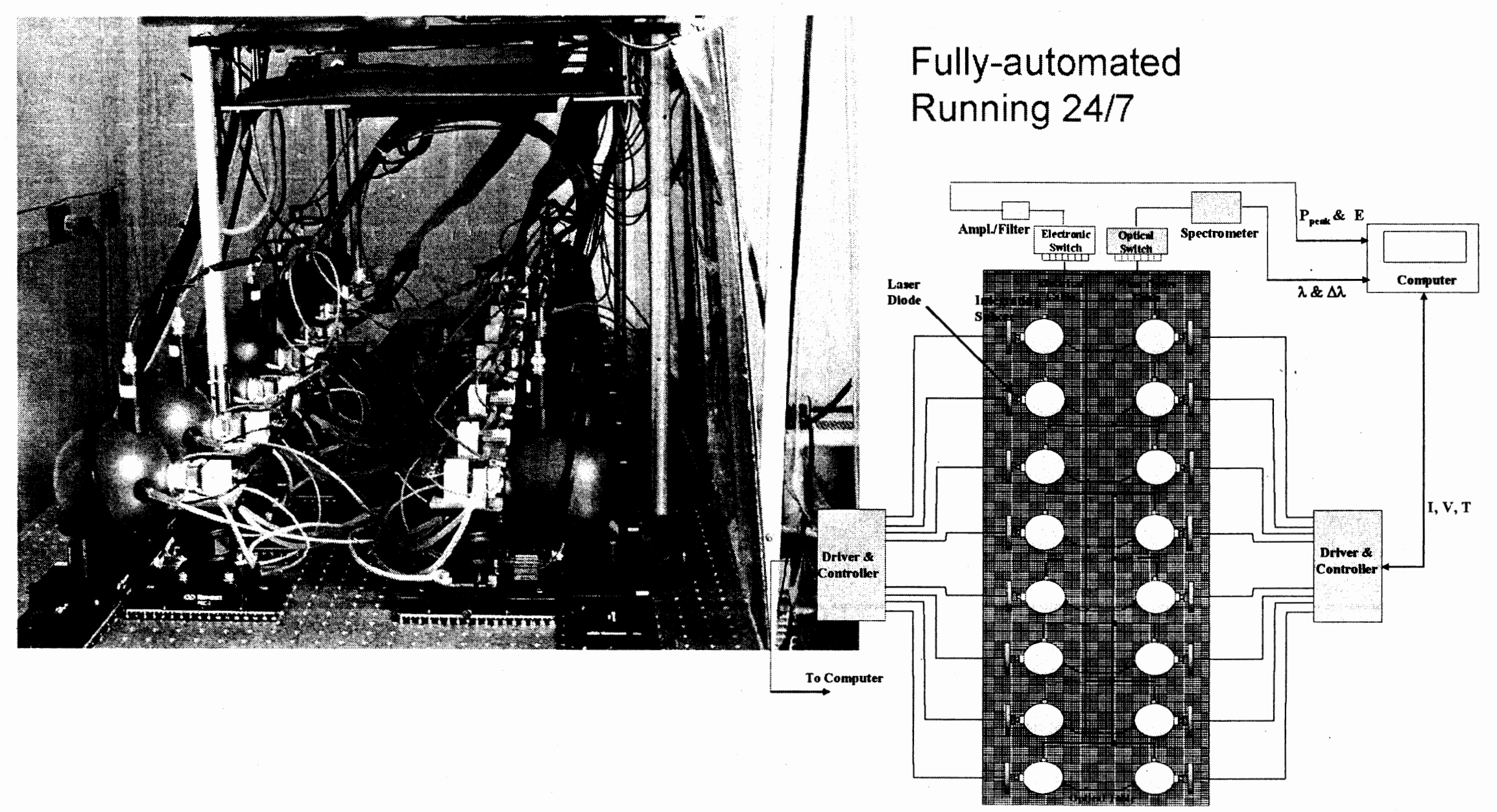




\section{Lifetime Testing of $792 \mathrm{~nm}$ LDAs Operating in Long Pulse Mode}

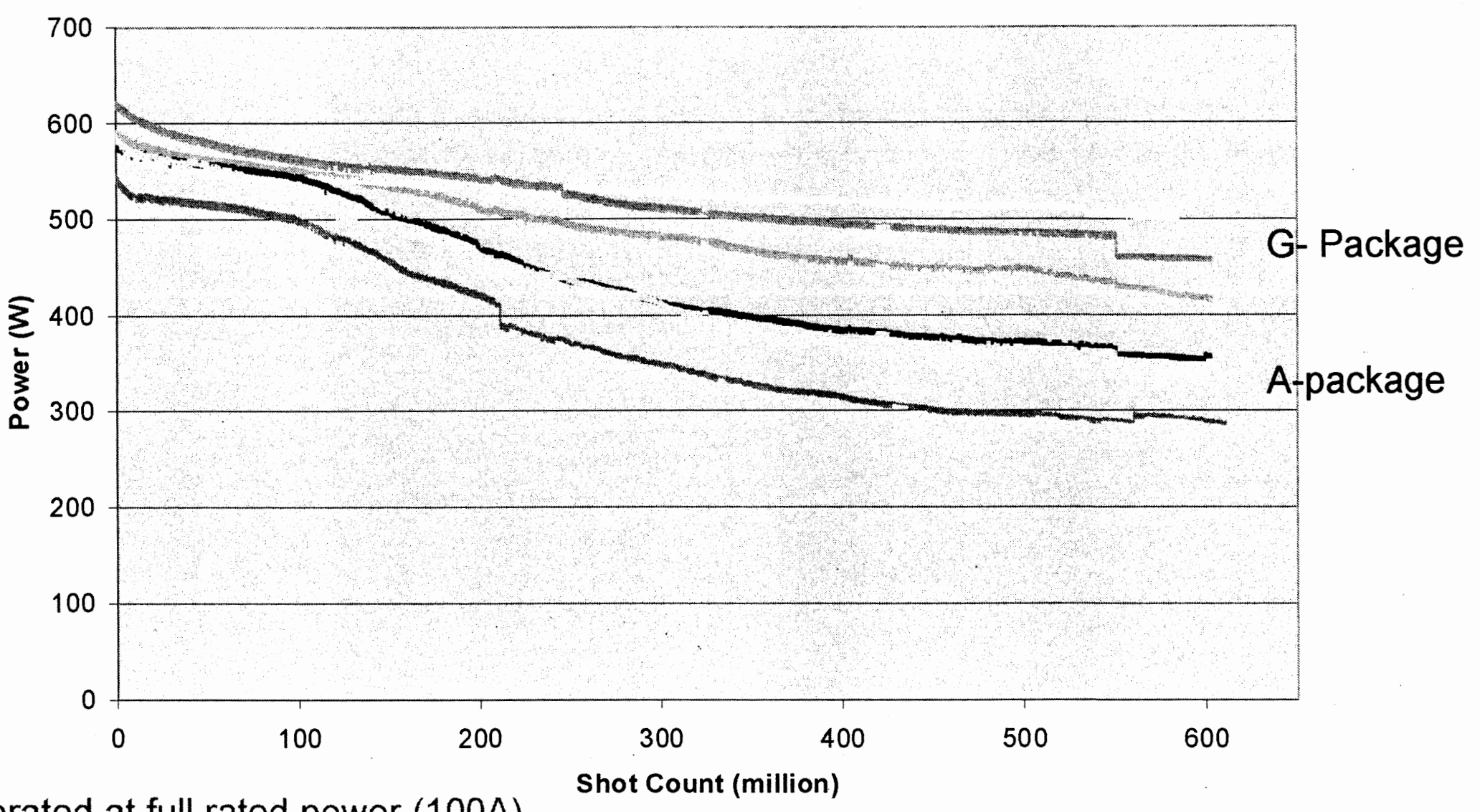

Operated at full rated power (100A)

Pulse repetition rate $=12 \mathrm{~Hz}$

Pulsewidth $=1 \mathrm{msec}$ 


\section{Improving Lifetime and Reliability of LDAs}

1. Evaluate and selection based on thermal characteristics and tolerance of thermomechanical stresses

\begin{tabular}{|l|l|l|}
\hline \multirow{4}{*}{ Package } & Geometry & A or G package style \\
\cline { 2 - 3 } & Architecture & Bars in Groove, Rack \& Stack, Stacked Subassemblies \\
\cline { 2 - 3 } & Heatsink Materials & BeO, $\mathrm{Cu}, \mathrm{CuW}$ \\
\hline \multirow{2}{*}{ Bar } & Efficiency & Wafer architecture and Epitaxy \\
\cline { 2 - 3 } & Fill factor & No. of emitters per bar \\
\hline
\end{tabular}

2. Specification based on trades between pump requirements and heat dissipation: number of bars and pitch size

3. Defining optimum operational parameters:

Drive current (de-rating), pulsewidth, pulse repetition rate, sink temperature

A package

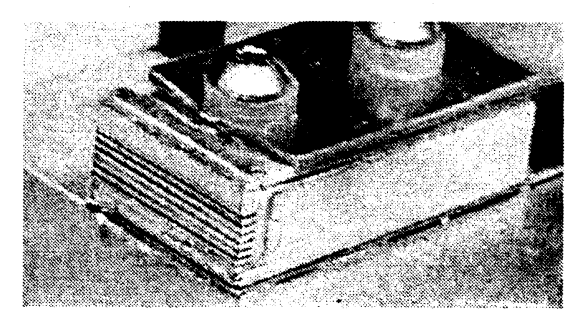

G package

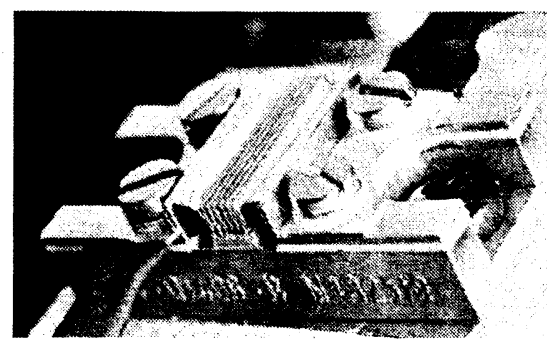




\section{Improving Lifetime and Reliability of LDAs}

Advancing Technology

\begin{tabular}{|l|c|}
\hline $\begin{array}{c}\text { Advanced package materials } \\
\text { (Composites, CVD Diamond) }\end{array}$ & $\begin{array}{c}\text { Higher thermal conductivity and } \\
\text { lower thermal mismatch }\end{array}$ \\
\hline Thin hard solder & Eliminate solder creep \\
\hline Smart Driver & Prevent current filamentation \\
\hline Integrated fuse & $\begin{array}{c}\text { Isolate damaged bars to allow for } \\
\text { continued operation of array }\end{array}$ \\
\hline Efficiency & Reduce generated heat \\
\hline
\end{tabular}

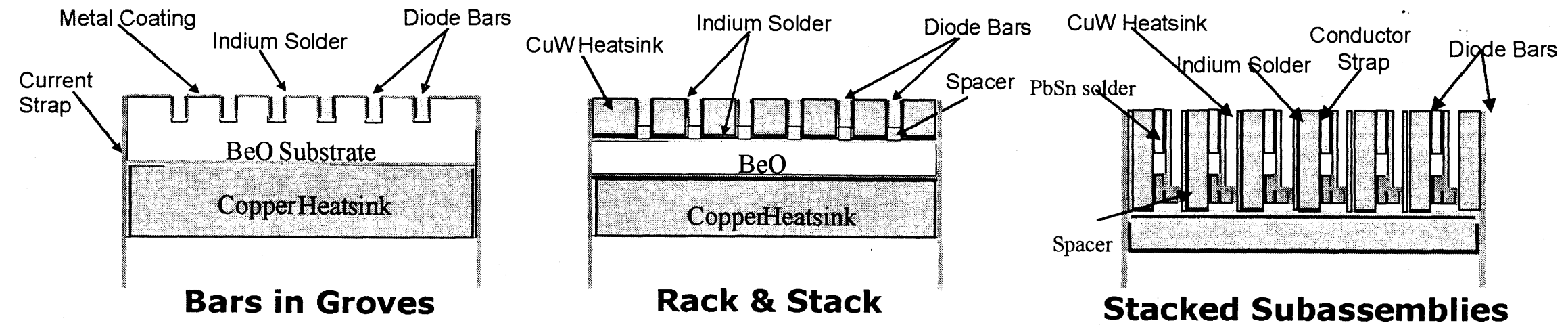


- Activity led by JPL and Raytheon

- Input provided by NASA LaRC and GSFC test programs

- Test program extended to JPL with additional contributions:

- Design of Experiments (DoE) back annotation for existing experiments

- Replication of test set-ups for validation

- Extension to single diode format for a large range of wavelengths corresponding to the diverse nature of laser applications at NASA

Note: this effort is partially funded under the NASA Electronic Parts and Packaging (NEPP) program from the Safety and Mission Assurance office. 


\section{DoE Overview}

\section{Purpose:}

- Establish common test methods

- Establish common procedures

- Review customer requirements to determine commonality

- Create a design of experiment (or a series of designs of experiment)

to optimize data collection and minimize cost and overlap

\section{Status:}

- Initial potential packaging failure modes identified

- Preliminary parameter list and ranges identified

- Preliminary response list selected

- Sample size and confidence limit trades bounded

- NASA requirements collated

- Common test set-up identified

- Preliminary designs identified

- Data collection for back-annotation started 


\section{Laser Packaging Failure Modes}

Some of the package failure modes encountered were:

- Au-In intermetallic formation at W/B

- Solder squeeze-out facet contamination

- General facet contamination

- Sn whisker

- Misalignment due to soft solders (In creep) 


\section{Experimental Parameters}

- Parameter lists

- Pulse integrated power (current varied with voltage measured)

- Pulse frequency: prf $0(\mathrm{cw})-40 \mathrm{kHz}$

- Pulse width: cw-30ns

- Responses

- Lifetime

- Number of pulses

- Time to 5, 10, 20 and $50 \%$ power loss 
Items being determined for the experiment:

- Thermal time constant for pulsed vs. CW operation

- Verification of test diode geometry (C-mounted devices initially)

- List of existing suppliers (completed)

- I/P list for supplier needs (completed)

- Accelerated testing

- Test set-up - configuration / distribution

- Test set-up - sampling 


\section{Conclusion}

- The National Diode-laser Array Working Group is approaching the end of its fourth year.

- Group facilitates focused interaction between the LDA user and provider communities.

- Group is authoring standards documents that address the specification and qualification of LDAs for operation in the space environment.

- NASA test and evaluation facilities are available to the community:

- 808-nm characterization and lifetest:

Nasir Kashem; 301-286-6360; nasir.b.kashem@nasa.gov

- 792-nm characterization and lifetest:

Farzin Amzajerdian; 757-864-1533; f.amzajerdian@nasa.gov

- Single diode lifetest (under construction):

Andrew Shapiro; 818-393-7311; aashapiro@jpl.nasa.gov 


\section{Working Group Schedule}

- Provisional meeting schedule:

- Friday, May 9, 2008; co-locate with CLEO (San Jose, CA), 6-8 May

- Tuesday, September 23, 2008; Penn. State U. Electro-Optics Center, Northpointe, Penn.

- Friday, January 30, 2009; Lockheed Martin, Sunnyvale, Calif. (immediately following Photonics West, January 24-29)

- For more info:

- Dave Tratt (The Aerospace Corp.) 626-395-7743

dtratt@aero.org

- Farzin Amzajerdian (NASA LaRC) 757-864-1533

f.amzajerdian@nasa.gov 